\title{
Study of Monoamine Oxidase-B and Indole Derivatives Using Two Molecular Docking Programs: Molegro and MOE
}

\author{
${ }^{1}$ W.Soufi ${ }^{*},{ }^{2}$ M. Merad, ${ }^{3}$ F. BOUKLI Hacene, ${ }^{4}$ S.Ghalem \\ ${ }^{1}$ Mascara University-AlGERIA \\ ${ }^{2,3,4}$ University of Tlemcen-AlGERIA \\ ${ }^{1}$ Faculty of science exact \\ ${ }^{1,2,3}$ Laboratory of Naturals Products and Bio actives-LASNABIO
}

\begin{abstract}
Inhibition of the enzyme Monoamine oxidase (MAO) is an important approach in the treatment of Parkinson's disease. A series of indole derivatives were synthesised and evaluated as inhibitors of MAO-B may give insight to develop new ways of antiparkinson drug, In general, the derivatives were found to be selective MAO-B inhibitors with IC50 values . MAO-B inhibitors, are considered useful in the therapy of Parkinson's disease since oxidation by MAO-B represents a major catabolic pathway of dopamine in the central nervous system .

Our goal of research is to study the inhibition of MAO-B by molecular modeling methods. Different molecular modeling tools are used to perform this work (molecular mechanics, molecular dynamics and molecular docking by two programms MDV ( molegro virtual docker) and MOE (modelling Opering Environment. The results obtained from this work, into which the inhibition of MAO-B by molecular modeling methods was elucidated, allow us to conclude that indole derivatives are promising reversible MAO-B inhibitors with a possible role in the treatment of neurodegenerative diseases such as Parkinson's disease (PD).
\end{abstract}

Keywords: Parkinson's disease (PD), Monoamine oxidase (MAO), indole derivatives, molecular modeling (Molegro, MOE).

\section{Introduction}

Neurodegenerative diseases of the human brain encompass a diversity of disorders. The age dependent neurodegenerative diseases include Parkinson's disease and Alzheimer's disease [1], which are caused by genetic and environmental influences and lead to the accumulation of protein aggregation thereby causing oxidative stress and inflammation. Parkinson's disease is characterized by progressive loss of dopamine producing neurons in the substantia nigra pars compacta, and results in a drastic depletion of dopamine in the striatum, to which these neurons project [2]. The discovery of PD (Parkinson's disease) genes has led to the hypothesis that misfolding of proteins and dysfunction of the ubiquitin-proteasome pathway are pivotal to PD pathogenesis [3]. Previously implicated culprits in PD neurodegeneration, mitochondrial dysfunction and oxidative stress, may also act in part by causing the accumulation of misfolded proteins, in addition to producing other deleterious events in dopaminergic neurons:

- Parkinson's disease begins after 70 years of various clinical signs of this disease occur in young people [4];

- a evolution to dementia and the occurrence of more frequent hallucinations;

- symptoms of dystonia, dyskinesia and motor fluctuations appear to be less frequent on youth health.

Monoamine Oxidase -B (MAO-B) is a mitochondrial protein located in the outer layer of the Mitochondria, it catalyses the metabolism of neurotransmitters such as dopamine[5,6]. MAO-B has therefore been of interest in the development of therapeutic agents for the treatment of MP [7,8]. Inhibition of MAO-B leads to an increase in dopamine levels and thus provides symptomatic relief in these patients [9]. MAO-B 
degrades dopamine and at the same time produces toxic byproducts such as ammonia hydrogen peroxide [10] and deprenyl [11], were one of the first therapeutic approaches developed to increase The bioavailability of dopamine and thus slow the evolution of this disease and still remain one of the treatments used.

Monoamine Oxidase Inhibitors (MAOIs) have therapeutic potency Potential [12]. Parkinson's MAO was divided into two subtypes [13], MAO-A and MAO-B. MAO-A inhibitors are prescribed for the treatment of depression and anxiety, MAO-B inhibitors are used with L-Dopa in the symptomatic treatment of PD.

\section{Materials and Methods}

\section{Enzyme structure}

The X-ray crystal structures of monoamine oxidase B was made from the data base Bookhaven Protein Data Bank (PDB ID: 2V5Z) [14], were downloaded from RCSB Database (www.rcsb.org/pdb) [15]. it is cocrystallized with the selective inhibitor safinamide (Figure 1).

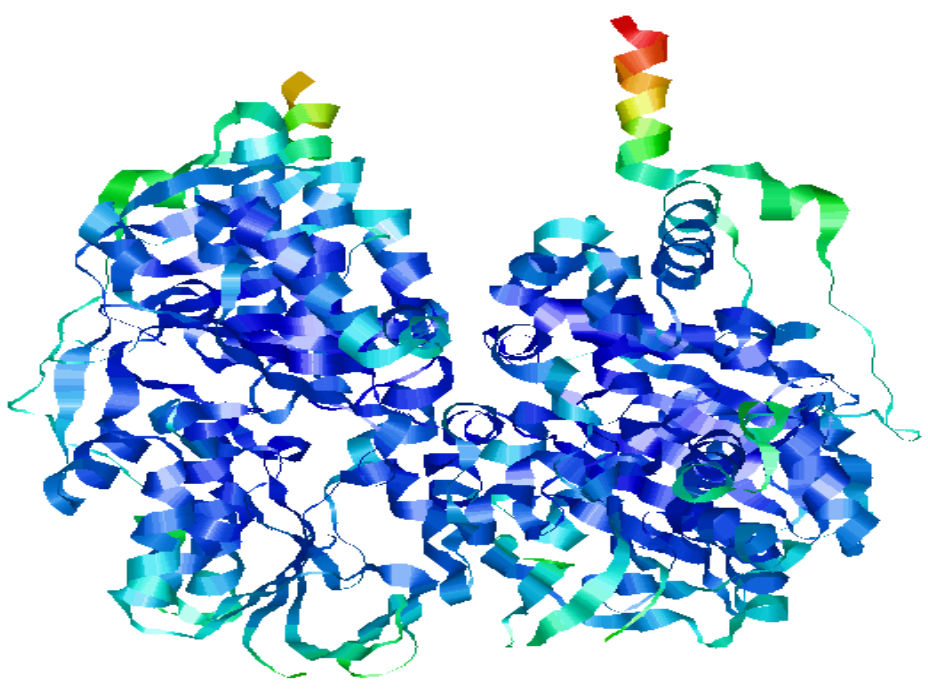

Figure 1: The dimer form of monoamine oxidase B

The three dimensional structure of monoamine oxidase B was obtained by X-ray diffraction with a resolution (1.60A).Note that the monoamine oxidase B crystallizes in the dimer form (Figure1), with 993 residues and 7911 atoms. With the reducing effect of molecular modelling, we simplified the model of the enzyme and retained only one monomer (Figure 2).

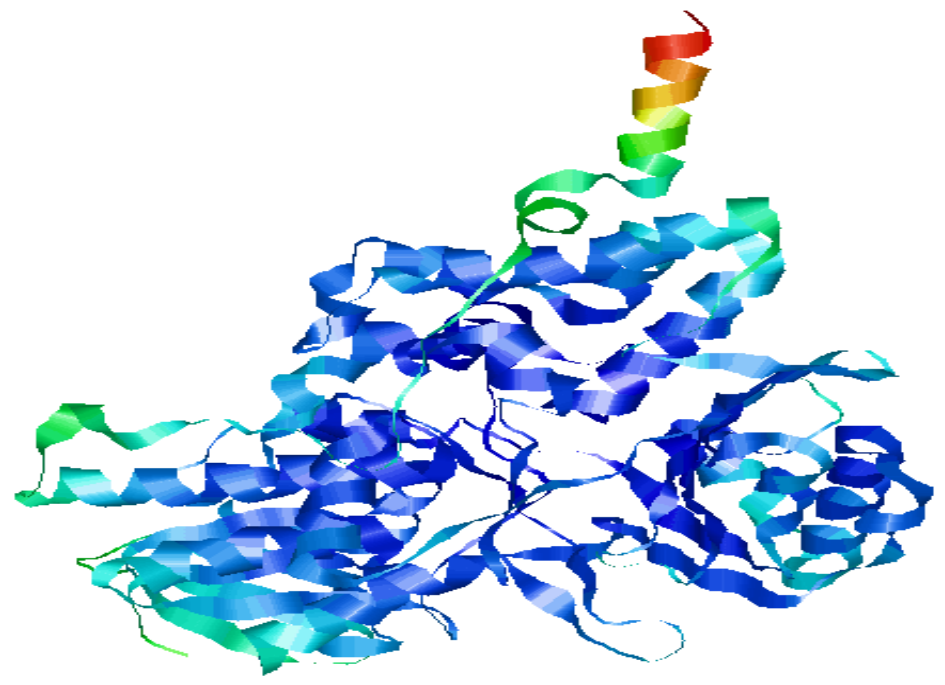

Figure 2: Simplified model of monoamine oxidase B 
Computational analysis was carried out on chain A of 2V5Z. The four inhibitor we used of indole derivatives which are synthesized [16], were selected to study the associated physico-chemical parameters and protein-ligands interactions.

As shown in figure 3 .The found structures were optimized by DFT level using hybrid functional density B3LYP with 6-31G basis (Gaussview 2.1).

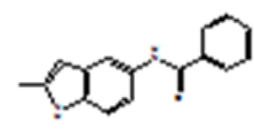

Inhibitor-1

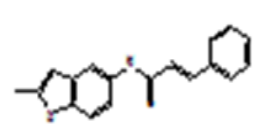

Inhibitor-2

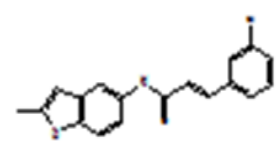

Inhibitor-3

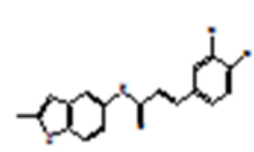

Inhibitor-4

Figure 3: indole derivatives

\section{Result and Discussion}

Molecular docking is an optimization problem, where the objective is to find the ligand binding mode with lowest potential energy. The process of docking involves sampling the coordinate space of the target binding site and scoring each possible ligand pose within that site, the highest scoring pose then taken as the predicted binding mode for that compound. There are many different docking programs now available and they differ in the nature of the sampling algorithms they employ, in their manner of handling ligand rigid and protein flexibility, the docking scoring functions they use of both programs Molegro Virtual Docker (MVD 2011)[17-18] and MOE software (modelling Opering Environment)[19].

MVD automatically identifies potential binding sites (also referred as cavities or active sites) by using its cavity detection algorithm. The cavities within a $30 \times 30 \times 30 \AA^{3}$ cube centered at the experimentally known ligand position were used. The cavities that are identified by the cavity detection algorithm are then used by the guided differential evolution search algorithm to focus the search, to that specific area during the docking simulation. In the case of the crystal structures for monoamine oxidase B complexes, the program generally identified five different binding sites (Figure 4). From these five predicted cavities the one with the highest volume $\left(222.208 \AA^{3}\right.$ ) was selected for consideration, as it includes the bound ligand.

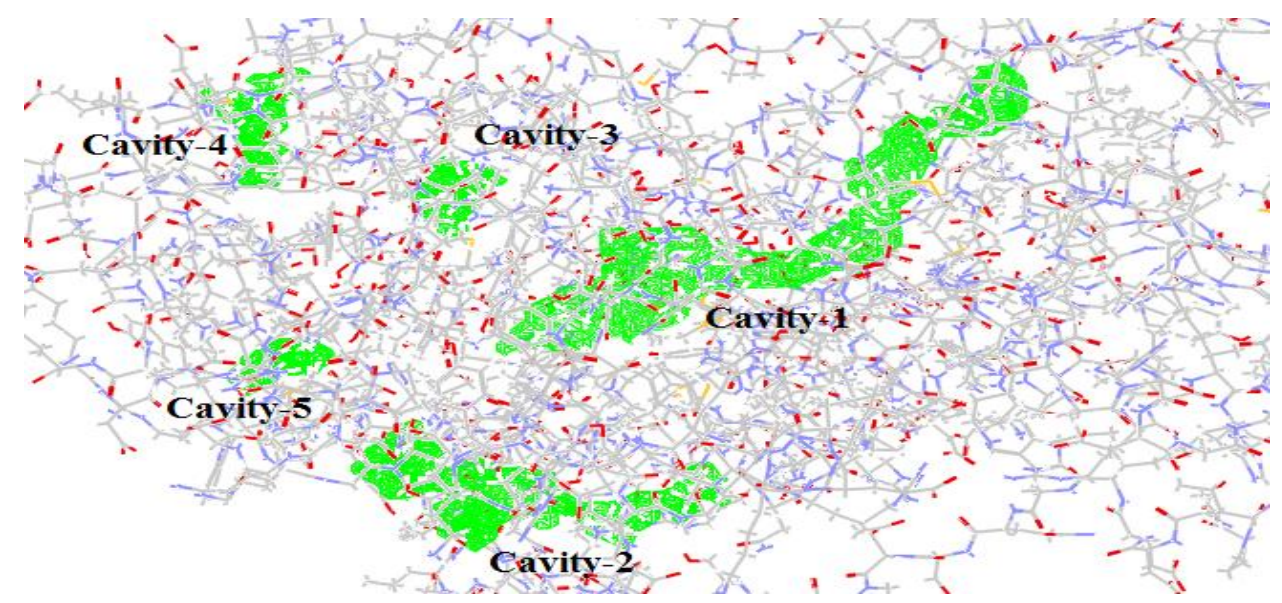

Figure 4: The five cavity MVD-detected cavities in monoamine oxidase-B, detected cavity green, carbon atoms grey, oxygen atoms red, nitrogen atoms blue.

MOE, Molecular Operating Environment, is a package that is to say a set Of several software programs for the discovery and computer-aided design of biologically active molecules. This allows several tasks to be performed for a very short time. It allows to draw the molecules, to minimize them to have the best conformations of these molecules. It also makes it possible to dock several ligands gathered in a data base in the active site of a protein in succession.

MOE has a flexible docking form in which the receptor and ligand changes conformation and adapt to the environment [20]. It is also possible to represent the enzyme and its co-crystalline ligand. 
Optimizing the geometry 2V5Z The enzyme was performed using the MMFF94x force field implemented in the MOE software (Molecular Operating Environment).

The main chain has been kept rigid, while the side chains remain flexible. This approximation allows the side chains of the proteins to find the position in which the interactions are most favorable.

The next step after the construction of ligands, and the positioning of these molecules in the active site of the enzyme (2V5Z). For this, we using of both programs Molegro [16-18] and MOE software (modelling Opering Environment)[19], the ligand-receptor complex is formed, it will adapt to the most stable conformation, i.e., the one with the lowest energy level.

The search for binding modes is generally constrained to a small specific region of the receptor called the active site. We then recorded the best score, that is, the one with the lowest energy corresponds to the best interactions between the ligand and the active site of the enzyme for of both programs Molegro and MOE software (modelling Opering Environment). The results are summarized in Table 1and Table 2 respectively.

Table 1: Docking results of Molegro (Kcal/mol)

\begin{tabular}{|l|l|l|l|l|}
\hline & Complexe -1 & Complexe -2 & Complexe -3 & Complexe -4 \\
\hline MolDock Score & -140.651 & -131.305 & -140.575 & -151.00 \\
\hline H bond $(\AA)$ & 4.0086 & 0 & -0.0619 & -0.3078 \\
\hline
\end{tabular}

Rescoring of these docked results using Molegro, revealed that inhibitor-1, inhibitor-2, and inhibitor-3 binds within the active site of MAO-B with binding free energy of $-140.651,-131.305$ and $-140.575 \mathrm{Kcal} / \mathrm{mol}$ respectively, that was higher than that found for inhibitor-4. $-151.007 \mathrm{Kcal} / \mathrm{mol}$. The binding mode of inhibitor-4 is within the active site of MAO-B is shown in Figure 5.
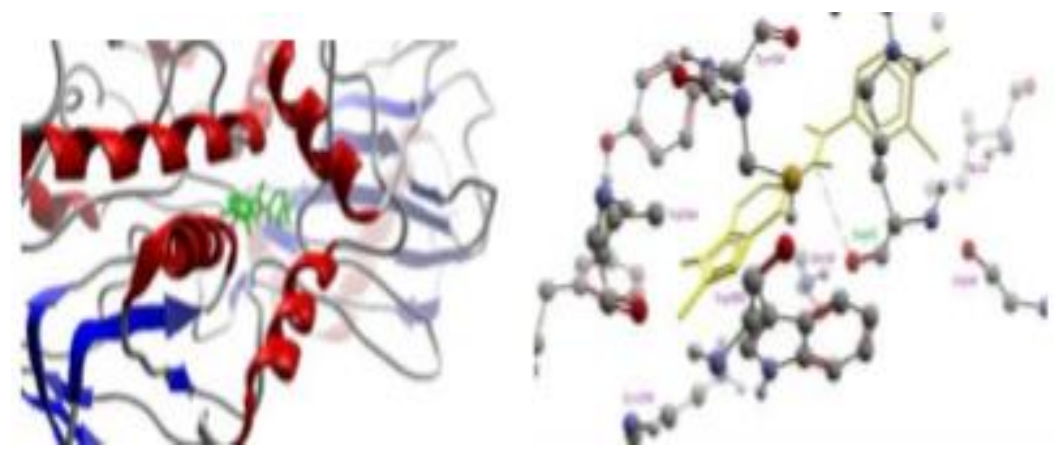

Figure 5: The predicted binding orientation of inhibitor-4 in the active site of MAO-B (2V5Z.pdb)

Table 2: Docking results of MOE (Kcal/mol)

\begin{tabular}{|l|l|l|l|l|}
\hline & Complexe -1 & Complexe -2 & Complexe -3 & Complexe -4 \\
\hline scoring & -7.2476 & -7.8561 & -7.9565 & -8.3335 \\
\hline Rmsd_Refine & 1.9991 & 1.7729 & 1.1551 & 1.0455 \\
\hline Number of poses & 8 & 8 & 8 & 7 \\
\hline
\end{tabular}

The following results revealed that complex 4 had the lowest energy $(-8.3335 \mathrm{Kcal} / \mathrm{mol})$ is more active than complex $3(-7.9565 \mathrm{Kcal} / \mathrm{mol})$, More active than complex $2(-7.8561 \mathrm{Kcal} / \mathrm{mol})$, more active than complex $1(-7.2476 \mathrm{Kcal} / \mathrm{mol})$.

A 2D molecular method of the screen has been assigned to the MOE (Molecular Operating Environment), which is designed to visualize the active sites of complexes (Protein-ligand) figure 6 . The ligand is arranged and rendered using an improved version of the $2 \mathrm{D}$ algorithm layout representation, And the protein residues are arranged around it to indicate spatial proximity bonds[21].

The interactions between $2.5 \AA$ and $3.5 \AA$ are considered high and those between $3.1 \AA$ and $3.55 \AA$ are assumed averages. Interactions greater than $3.55 \AA$ are weak or absent [22]. 

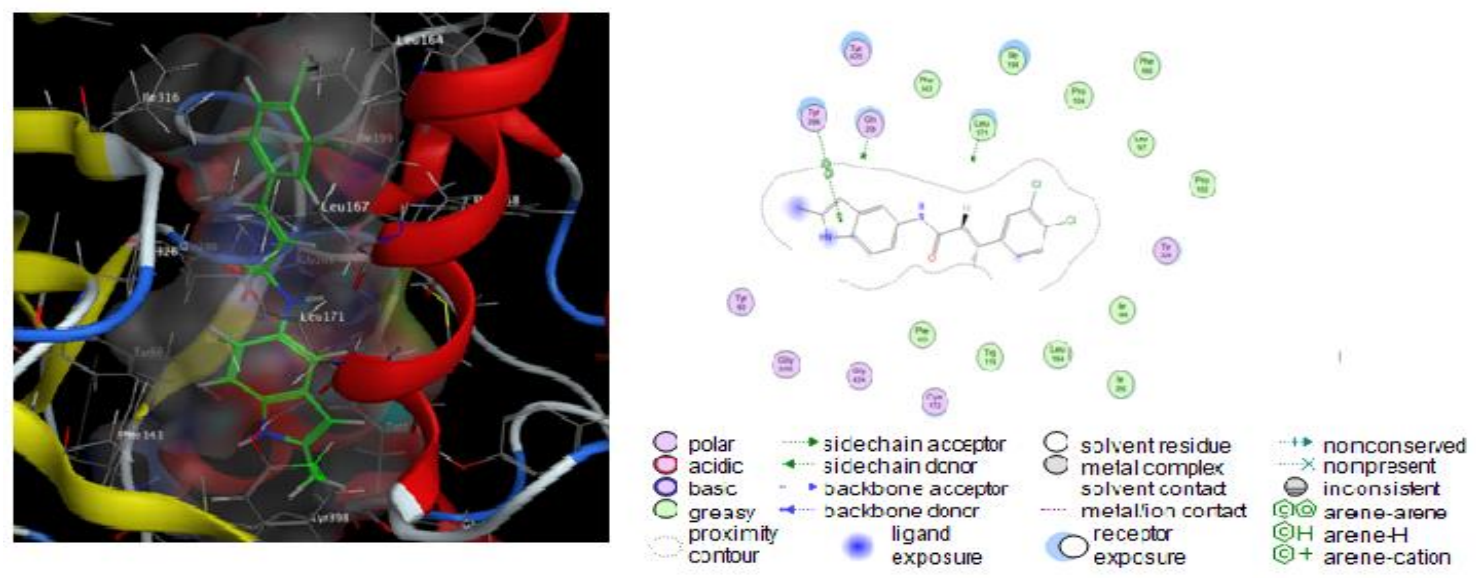

Figure 6: Docking of inhibitor-4 in the cavity

\section{Ligand Interactions Report}

Mon Jun 05 14:33:58 2017 (MOE 2014.09)

2V5Z: OXIDOREDUCTASE / 2V5Z: OXIDOREDUCTASE

\begin{tabular}{|c|c|c|c|c|c|c|c|}
\hline igand & $\operatorname{Re}$ & & & & Interaction & Distance & $\mathrm{E}(\mathrm{kcal} / \mathrm{mol})$ \\
\hline N21 37 & OE1 & GLN & 206 & (A) & $\mathrm{H}$-donor & 2.79 & -3.1 \\
\hline 6-ring & CG & LEU & 171 & (A) & pi-H & 3.74 & -0.6 \\
\hline
\end{tabular}

The results obtained using the two approaches (Molegro and MOE software) reveal that inhibitor-4 is a good inhibitor for $2 \mathrm{~V} 5 \mathrm{Z}$ enzyme, so it appears that the inhibitor-4 presents probably a better contribution to inhibition by other to slow the progression of Parkinson's disease.

The IC50 value of the inhibitor with the co-crystallization is SAG $0.08 \mu \mathrm{M}$. The reliability of the inhibitor -4 was confirmed by comparing the experimental values of IC 50 in Table 3 with the IC50 value of the inhibitor co- crystallization SAG so we can say that the inhibitor-4 the smallest value of IC $50=0.06 \pm$ $0.01 \mu \mathrm{M}$, inhibitor-4 thus has a better inhibition compared to other inhibitors.

Table 3: Experimental IC50 values of various ligands [14]:

\begin{tabular}{|l|l|}
\hline Compounds & $\mathrm{IC}_{50}(\mu \mathrm{M})$ \\
\hline Inhibitor-1 & $3.84 \pm 0.75$ \\
\hline Inhibitor-2 & $18.82 \pm 3.28$ \\
\hline Inhibitor-3 & $9.83 \pm 1.63$ \\
\hline Inhibit0r-4 & $0.06 \pm 0.01$ \\
\hline
\end{tabular}

\section{Conclusion}

The docking studies as described above provide estimation on inhibitory activities of the docked ligand. The results showed that inhibitor-4 fits well in the active site of MAO-B and also interact with the residues in the active site which are important for their biological activity.

After comparing the results of the theoretical study of the inhibition of monoamine oxidase B and those of the experimental study, we see that the two results are consistent for inhibitor-4.Further, work can be extended to study the receptor-ligand interactions experimentally and evaluation of their biological activity would help in designing compounds based on virtual screening techniques. In this study we have demonstrated that of inhibitor-4 is important for MAO-B activity and this information may give insight to develop a novel antiparkinson drug. 


\section{Reference}

[1.] Aruoma, O. I.; Bahorun, T.; Jen, L. S. Neuroprotection by Bioactive Components in Medicinal and Food Plant Extracts. Mutation Research 2003, 544, 203-215.

[2.] Lotharius, J.; Brundin, P. Pathogenesis of Parkinson's Disease: Dopamine, Vesicles and $\alpha$ Synuclein. Nature Reviews-Neuroscience 2002, 3, 1-10.

[3.] William, D.; Serge, P. Parkinson's Disease: Mechanisms and Models. Neuron 2003, 39, 889-909.

[4.] Roze, E. What Are the Therapeutic Options for Parkinson's Disease in Elderly?. Cah. 2 Year Gerontol. 2012, 4, 50-55.

[5.] Edmondson D. E, Mattevi A, Binda C, Li M, Hubalek F. Curr. Med. Chem, 2004,11, 1983.

[6.] Binda C, Hubalek F, Li M, Edmondson D. E, Mattevi A. FEBS Lett, 2004, 564, 225.

[7.] Youdim M. B, Edmondson D, Tipton K. F. Nat. Rev. Neurosci, 2006, 7, 295.11.

[8.] Youdim M. B, Geldenhuys W. J, Van der Schyf C. J. Parkinsonism Relat. Disord, 2007,13, S281.

[9.] Fernandez H. H, Chen, J. J. Pharmacotherapy, 2007, 27, 174S.

[10.] Bortolato M, Chen K, Shih J. C. Adv. Drug Delivery Rev, 2008, 60, 1527.

[11.] Coatrieux C. Monoamine oxydases et athérosclérose : signalisation mitogène et études in vivo. Thèse de doctorat en Discipline : Innovation Pharmacologique. Toulouse : Université Toulouse III - Paul Sabatier. France. 2007, 14p.

[12.] Ramsay RR, Gravestock MB. Monoamine oxidases: to inhibit or not to inhibit. Mini Rev Med Chem, 2003, 3, 129-136.

[13.] Li-Hua Mua, Bo Wanga, Hao-Yang Ren, Ping Liu, Dai-Hong Guo, Fu-Meng Wang, Lin Bai, YanShen Guo. Synthesis and inhibitory effect of piperine derivates on monoamine oxidase. Bioorganic \& Medicinal Chemistry Letters, 2012, 22 , 3343-3348.

[14.] L. H.A. Prins, J. P. Petzer, S. F. Malan. Inhibition of monoamine oxidase by indole and benzofuran derivatives. European Journal of Medicinal Chemistry ,2010, 45,4458-4466.

[15.] Thomsen R, Christensen M. H. J Med Chem , 2006 , 49(11), 3315-3321.

[16.] E. F. F. da Cunha, R. C. A. Martins, M. G. Albuquerque, J. Mol. Mod ., 2004 , 10, 297-304.

[17.] F. F. da. Elaine, F. B. Edilaine, A. O. Aline, C. R. Teodorico, J. Biom. Struct Dyn ., 2010 , 27, 619-625.

[18.] T. C. Ramalho, M. V. J. Rocha, E. F. F. da Cunha, M. P. Freitas, Expert Opin. Ther. Pat ., 2009 , $19,1193-1228$.

[19.] Molecular Operating Environment (MOE) Chemical Computing Group, Montreal, Quebec, Canada10, 2012.

[20.] (a) A.M. Clark,P.Labute; M. Santavy, "2D Structure Depiction", J. Chem.46, Inf. Model 2006.P 1107-1123; (b) Chemical Computing Group Journal 2005.

[21.] A.M. Clark, P.Labute.Detection and Assignment of Common Scaffolds in Project Databases of Lead Molecules; J. Med. Chem. 52, P469-483.2008.

[22.] Ayachi, H.; Merad, M.; Ghalem, S. Study of Interaction between Dipeptidyl Peptidase-4 and Products Extracted from the Stevia Plant by Molecular Modeling. International Journal of Pharmaceutical Sciences Review and Research 2013, 18, 87-90. 